\title{
Optimal power flow with valve point loading effects of cost function and mixed-integer control variables using big-bang and big-crunch optimization
}

\author{
C. V. Gopala Krishna Rao*, G. Yesuratnam \\ Associate professor, E.E.E. Dept., M.V.S.R Engineering College, Hyderabad \\ Associate Professor, Head, EE Dept., University college of Engineering, Osmania University, Hyderabad \\ *Corresponding author E-mail: vgkrao_ch@yahoomail.com
}

\begin{abstract}
This paper presents application of Big Bang and Big crunch(BB-BC) a nature inspired optimization method which is developed from the concepts of universal evolution to solve complex static optimal power flow (OPF) with an aim to obtain minimum cost of thermal power generating units whose cost functions are non-convex due to valve point loading effects. Control variables to optimize cost functions by satisfying usual constraints of OPF are of continuous and discrete type (mixed- integer control variables). Mathematical programming approaches presents problem in solving non-convex OPF. Nature inspired heuristic methods can be applied to solve such non-convex optimization problems. One of the requirements of heuristic methods is numerical simplicity without trial parameters in update equation of optimization along with reliability and ease in developing computer code for implementation. Most of the nature inspired methods search efficiency and reliability depends on choice of trial parameters to update control variables as optimization advances in search of optimal control variables.BB-BC optimization has search ability on par with other popular heuristic methods but free from choice of trial parameters is applied to obtain OPF solutions on two typical power systems networks and results are compared with MATLAB-7.0 pattern random search optimization tool box .Digital simulation results indicates a promising nature of the BB-BC to deal with non-convex optimization requirements of power system situations .
\end{abstract}

Keywords: Bing-Optimal power flow (OPF), Bang and Big-Crunch, valve point loading effects.

NOTATIONS : $F_{T}$ : total operating cost, NB: number of buses, NG: number of generator buses, NT: number of Transformers, NL: number of lines (branches),t: tap position, NPQ: number of load buses NTR: number of transformers, $P_{i}$ : active power injection at bus i, $Q_{i}$ : reactive power at bus i,NP: population size (number of Big-Bangs BB-BC),NC: number of control variables (dispersions in BB-BC), k:Generation/iterations of optimization,

\section{Introduction}

Rapid growth in power system size and Electrical power demand, problem of reducing the operating cost has gained importance while maintaining voltage security and thermal limits of transmission line branches. A large number of mathematical programming(algorithm)and AI (Artificial Intelligence technique ) have been applied to solve OPF ${ }^{[1,2]}$.In most general formulation, the OPF is a nonlinear, non-convex, large scale, static optimization problem with both continuous and discrete control Variables. Mathematical programming approaches such as calculus methods, Nonlinear programming (NLP), Linear programming (LP), Quadratic programming (QP), algorithms applied to obtain OPF solution require smooth and continuous cost function. OPF involving non-convex objective functions with mixed integer

Control variables further increases the complexity and presents problem to mathematical approaches. Dynamic programming methods (DP) are good at solving quadratic and ramp cost functions, at the cost of increased dimensionality and may get struck in local optimality ${ }^{[3]}$. Recent advances in AI techniques can be applied as complementary approach to pave the way towards global/near global solutions for complex optimization problems such as $\mathrm{OPF}{ }^{[2]}$. All search intelligence techniques, are population based, stochastic in nature offers multiple solutions (per iteration/generation) are developed by scientific community from the inspiration of natural social behaviour of different 
organisms/ and natural Processes. Genetic algorithms and its variants, Swarm intelligence, Bacteria foraging, ant-colony search techniques are applied to obtain quality solutions [4] to optimization problems. These heuristic optimization algorithms require appropriate tuning parameters to arrive at better quality solutions to avoid algorithms being trapped into local optimality.

Big-Bang and Big-Crunch (BB-BC) developed by Erol and Eksin [5] from the concept of universal Evolution, is also a population based search technique. BB-BC method has been proved to outperform Genetic algorithm for bench mark test functions [5]. BB-BC is numerically a simple optimization method with very few optimization parameters and further algorithm does not involve trial parameters for optimal search. This paper aims at solving complex OPF problem with an objective of minimizing Fuel cost of real power generating units , whose cost functions are considered as nonsmooth i.e with valve point loading effects. The control Variables considered to optimize the cost function are continuous and discrete variables. Continuous variables are Generator real power outputs and Generator terminal voltages. Discontinuous variables are transformer tap settings.

As BB-BC picks up Control variables from random population, the algorithm is run ten times with different random initial Control variablesto have an idea of spread of cost statistics such as best cost, worst cost and mean cost. The Results are compared with commercially available MATLAB adoptive pattern search tool-box optimization [ 6] to have an idea of accuracy of final results. Reason for choosing pattern search random optimization is that it is also a simple optimization method without any tuning parameters. Best results out of 10 run simulation is presented for the both optimization methods, along with a comparative summary of OPF results.

\section{Cost curve of generating units}

In general fuel cost of thermal power generating units is approximated as quadratic cost function of real power outputs. Thus the total cost of all units involved in real power generation is as follows.

$$
F_{T}\left(p_{g}\right)=\sum_{i=1}^{N g} a_{i}+b_{i} P_{g i}+c_{i} P_{g i}^{2} \$ / \mathrm{h}
$$

where $a_{i}, b_{i}$, and $c_{i}$ are cost coefficients. When each steam admission valve in turbine starts to open and at the same time a rippling effect on the unit curve is produced, this effect can be modelled as rectified sinusoidal contribution to the quadratic cost functions ${ }^{[7]}$. Therefore, with valve point loading effects total cost of real power generation is as given below.

$$
F_{T}\left(p_{g}\right)=\sum_{i=1}^{N g}\left[a_{i}+b_{i} P_{g i}+c_{i} P_{g i}^{2}+\left|d_{i} \times \sin \left\{e_{i} \times\left(P_{g i}^{\min }-P_{g i}\right)\right\}\right|\right]
$$

where $\mathrm{d}_{\mathrm{i}}, \mathrm{e}_{\mathrm{i}}$ are valve point coefficients, $P_{g i}^{\min }$ and $P_{g i}^{\max }$ are lower and upper power generation limits of thermal generating units .In this paper equation (2) is considered as objective function in OPF .

\subsection{Problem formulation}

The goal of optimal power flow is to determine the optimal settings of real power output of generating units, terminal voltages of generating units and transformer tap settings i.e vector of feasible control variables $u$ while maintaining several constraints of power system network leading to minimum cost $\left(F_{t}\right)$ of thermal power generating units.OPF problem can be mathematically stated as follows .

$$
\min _{\mathrm{t}}\left(\mathrm{p}_{\mathrm{g}}\right)=\sum_{i=1}^{N g}\left[a_{i}+b_{i} P_{g i}+c_{i} P_{g i}^{2}+\mid d_{i} \times \sin \left\{e_{i} \times\left(P_{g i}^{\min }-P_{g i}\right)\right\}\right]
$$

Subjected to

a) Bus real and reactive power balance equality constraints

Real power balance in the network

$$
P_{i}(\mathrm{~V}, \delta)-P_{g i}+P_{d i}=0 \quad(\mathrm{i}=1,2,3, \ldots \mathrm{NB})
$$


Reactive power balance in the network

$$
Q_{i}(\mathrm{~V}, \delta)-Q_{g i}+Q_{d i}=0 \quad(\mathrm{i}=1,2, \ldots \mathrm{NPQ})
$$

Where $\mathrm{V}, \delta$ are magnitude of bus voltages and bus angles.

b) inequality operational constraints of power system network

(i) limits on reactive power generation of generator buses

$$
Q_{g i}^{\min } \leq Q_{g i} \leq Q_{g i}^{\max }
$$

(ii) limits on voltage magnitudes of load buses(PQ)

$$
V_{i}^{\min } \leq V_{i} \leq V_{i}^{\max }
$$

(iii) thermal limits of transmission lines

$$
S_{i} \leq S_{i}^{\max }
$$$$
(\mathrm{i}=1, \ldots \mathrm{NL})
$$

Inequality constraints of feasible control variables are randomly generated while the inequality operational constraints and slack generator real power output are handled by a quadratic penalty function approach .Due to inclusion of penalty terms equation (3) transforms to a pseudo objective function (FF) .

$$
\min F F=F_{T}\left(P_{g}\right)+P_{S}+P_{v i}+P_{q i}+P_{L i}
$$

where $P_{s}, P_{v i}, P_{q i}$ and $P_{L i}$ are as follows

$$
\begin{aligned}
& P_{s}=P_{e n s}\left(P_{g s}-P_{g s}^{l m t}\right)^{2}, \text { where } \quad P_{g s}^{l m t}=\left\{\begin{array}{l}
P_{g s}^{\min } ; \text { if } P_{g s}<P_{g s}^{\min } \\
P_{g s}^{\max } ; \text { if } P_{g s}>P_{g s}^{\max }
\end{array}\right. \\
& P_{v i}=P_{e n v} \sum_{i=1}^{N P Q}\left(V_{i}-V_{i}^{l m t}\right)^{2}, \text { where } V_{i}^{l m t}=\left\{\begin{array}{l}
V_{i}^{\text {min }} ; \text { if } V_{i}<V_{i}^{\text {min }} \\
V_{i}^{\text {max }} ; \text { if } V_{i}>V_{i}^{\max }
\end{array}\right. \\
& P_{q i}=P_{e n q} \sum_{i=1}^{N_{g}}\left(Q_{g i}-Q_{g i}^{l m t}\right)^{2} \quad, \text { where } Q_{g i}^{l m t}=\left\{\begin{array}{l}
Q_{g i}^{\min } ; \text { if } Q_{g i}<Q_{g i}^{\min } \\
Q_{g i}^{\max } ; \text { if } Q_{g i}>Q_{g i}^{\max }
\end{array}\right. \\
& P_{L i}=P_{\text {enMVA }} \sum_{i=1}^{\text {Nline }}\left(S_{i}-S_{i}^{l m t}\right)^{2}
\end{aligned}
$$

here $P_{e n s}, P_{e n v}, P_{e n q}, P_{\text {enMVA }}$ are penalty values for the slack bus generator MW limit violation, Load bus voltage limit violations, generator reactive power limit violations and violations for thermal limits of lines respectively.

\section{Big-bang and big-crunch (BB-BC)}

Big-Bang and Big-crunch (BB-BC) optimization, is developed from the concept of universal evolution. Big-Bang Phase relates to energy dispersion in random state before evolution of universe. The dispersed energy is drawn into an order for the formation of universe. The stage of drawing the energy to an ordered state is Big-crunch phase. This concept can be mathematically simulated by obtaining object function values by creating random control variables (Big -Bang) phase. The Centre of Mass (CM) of Big-Bang phase is drawn into an ordered state by a Big- crunch phase. Crunch phase control variables emerge as best control variables from Big-Bang phase. Sequential repetition of Big-Bang around $\mathrm{CM}$ eventually leads to the global control variables of the function to be optimized. In the Big Bang phase control matrix $(\mathrm{U})$ of dimension $(\mathrm{NP} * \mathrm{NC})$ is generated within lower and upper limits of control variables. Each row of control variable is substituted in function to be optimized to obtain NP number of function values. Then centre of mass $u_{C M}$ of first phase dispersions can be computed using equation 5.

$$
u_{C M}=\sum_{i=1}^{N C}\left(1 / f^{i}\right) * u^{i} /\left(\sum_{i=1}^{N C} 1 / f^{i}\right)
$$


Computation of $u_{C M}$ is crunch phase of the optimization. In equation $5, u^{i}$ is $\mathrm{i}^{\text {th }}$ row of $\mathrm{U} . f^{i}$ is the function value corresponding to $u^{i}$. This completes $\mathrm{k}^{\text {th }}$ generation of optimization method. For $(\mathrm{k}+1)^{\text {th }}$ generation, each row of control vector is updated around $u_{C M}$ using update the following update equation.

$$
u^{i}=u_{C M}+\left(u^{l m t} * \operatorname{randn}\right) / k
$$

where $u^{l m t}$ is scale of upper $\mathrm{U}^{\text {upper }}$ and lower $\mathrm{U}^{\text {lower }}$ limits of the control variables, $\mathrm{K}$ is generation number, "randn" is normally distributed random number between -1 and +1 , which changes for each control variable. Repetition of Big Bang followed by crunch results in optimum value of the function. From the general explanation of BB-BC it is clear that like all population search methods, BB-BC also initiates search from random initial variables, and starts exploring better values of objective function using an update rule governed by equation (6).The update equation that Controls optimization process does not contain any trial parameters as is usually the case in many population search methods. This is an attractive feature of BB-BC that makes the algorithm numerically simple.

\subsection{Steps to implement BB-BC to Power system optimization}

1. Read OPF data (cost coefficients of objective function, Emission coefficients, Line, bus data and location of control variables) in power system network.

2. Generate initial control variable matrix $\mathrm{U}$ of size $(\mathrm{NP} * \mathrm{NC})$ within the lower and upper

limit of control variables i.e $\mathrm{i}^{\text {th }}$ row of $\mathrm{U}$ can be generated as

$$
\mathrm{u}^{\mathrm{i}}=\mathrm{U}^{\text {lower }} *\left(\mathrm{U}^{\text {upper }}-\mathrm{U}^{\text {lower }}\right) * \text { rand }(1, \mathrm{NC}) .
$$

Where, 'rand' is uniform random number $[0,1]$, $\mathrm{U}^{\text {lower }}$ and $\mathrm{U}^{\text {upper }}$ are lower and upper limits of control variables respectively . Typically, $\mathrm{U}^{\text {lower }}$ and $\mathrm{U}^{\text {upper }}$ are row vectors of dimension $(1 * \mathrm{NC})$.

3. Set generation(iteration) count $\mathrm{k}=1$.

4. Initialize FF count to 1 . Row select of $U$ to 1 .

5. Fetch the row corresponding to Row select from U, modify line and bus data of power system network. Solve for real and reactive power balance equation by using Newton Raphson (NR)/FDLF ${ }^{[8]}$ and obtain solution for magnitude of load bus bar voltages $(\mathrm{V})$ and bus angles $(\delta)$ for all bus bars with slack bus as reference angle. calculate inequality constraints.

6. Check for inequality constraints, for any violation of these constraints, activate penalties and Evaluate FF. set Row select=Row select $+1, \mathrm{FF}=\mathrm{FF}+1$, return to 5 , till $\mathrm{FF}$ count=NP.

7. Obtain $u_{C M}$ using equation 4.check for stopping criteria, if met display current best solution, else go to step 8 .

8. Update control variables in accordance with update equation [6]. This step may result in violation of control variable limits. Those violated control variables should be made equal to their respective violated limit.

9. Set $\mathrm{k}=\mathrm{k}+1$.Return to step 3 till $\mathrm{k}=$ Maximum generations.

power balance equations (step 5), are solved by Newton Raphson power flow (NRLF) with real and reactive power balance equations to mismatch tolerance $0.0001 \mathrm{pu}$. Convergence criteria may be number of generations or difference between best function value of $\mathrm{k}^{\text {th }}$ and $(\mathrm{k}+1)^{\text {th }}$ generation less than a specified tolerance. The above steps are implemented for the two test systems mentioned in this paper. The required code is written in MATLAB-7.0, as m-files using library routines of MATLAB 7.0 soft ware. Code is executed on a $2.1 \mathrm{GHz}$, Pentium IV PC. 


\section{Simulation results.}

Test System 1 is Gangour -25 [9] bus system with 35 transmission lines, 5 generators. Cost coefficients are provided in Appendix. Total base case load of the system $(7.3 \mathrm{pu}+\mathrm{j} 2.23 \mathrm{pu})$ on $100 \mathrm{MVA}$ base. Total control variables are ten real valued continuous variables pertaining to five generating units real power output and terminal voltages of the generating units. Valve point loading effects are considered for all five real power output thermal generating units. Test System 2 is IEEE-30 [10] bus system with 41 transmission lines, 6 generators . Cost coefficients are provided in Appendix. For this case valve point loading effect is considered for first two real power out units. Total base case load of the system $(2.834 \mathrm{pu}+\mathrm{j} 1.26 \mathrm{pu})$ on 100MVA base. Total control variables are 16, 12 real valued continuous variables pertaining to six generating units real power output and terminal voltages of the generating units and four tap changing transformers whose tap positions are integer values. Capacitive shunts at bus number 10 and 24 are fixed at $0.19 \mathrm{pu}$ and $0.043 \mathrm{pu}$ respectively. Both the system slack bus is bus no1, whose real power output is constrained within the limits by penalty factor approach. Population size (NP) is kept at 40 and 50 for test case-1 and test case- 2 respectively. The Penalty values $P_{\text {ens }}, P_{\text {env }}, P_{\text {enq }}$, and $P_{\text {enmva }}$ for violations operational OPF limits are 1000, 10, 10, and 100 respectively . Load bus voltage limits are $0.95 \mathrm{pu}$ lower limit and $1.05 \mathrm{pu}$ upper limit. Terminal voltage limits of generating units are $0.9 \mathrm{pu}$ to 1.1 pu.For test case 2, 0-40 integer Steps, with step size 0.005 are considered for tap positions of tap changing Transformers. Minimum tap position ' 0 ' effects a tap of $0.9 \mathrm{pu}$ while maximum tap position ' 40 ', effects a tap of $1.1 \mathrm{pu}$. Pattern search tool box in MATLAB, is a random search method that searches a better function value in the neighbourhood of current best function value by creating search variables called meshes. Pattern search can be invoked in MATLAB environment by function name 'patterensearch'. This function accepts address of function to be optimized, lower ,upper bounds of the control variables and number of objective function evaluation, tolerance of mesh size, and options to plot the progress of optimization as arguments and returns the objective function values ,history of optimization which also includes reduction in mesh sizes.OPF problem is also solved in this paper by pattern search. Total function evaluation are set to 1200 and 1500 for test case-1 and 2 respectively as BB-BC is also evaluated for same number of function evaluations. Mesh size is set to adoptive mode for dynamic search process. Penalty terms are also same as mentioned above for BB-BC. Out of 10 run simulation with each run kept for maximum number of objective function computations the best control variables are indicated in Table-1. Final cost, losses, magnitude of load bus voltage distribution and maximum flow in a critical line is shown in Table-2.From these results itTable-1: Best control variables for testcase-1and test case- 2 .

is clear that for test case-1 real power outputs by both optimizations are almost same but in case-2 BB-BC has resulted in best control variables. Both optimization methods provides voltage profiles of load buses between $0.95 \mathrm{pu}$ to $1.05 \mathrm{pu}$ within the thermal limits of lines. For all transmission lines power flows by both optimization methods maintained good margins, however critically loaded lines and their thermal limits are indicated in table-2.Table-3 indicates statistics for 10 runs with different initial values. For test case 1, worst cost occurred only one time out of ten run for both optimization methods. For both test cases the mean cost provided by BB-BC is much superior to pattern search optimization. This indicates the reliability of BB-BC in arriving at optimal solution irrespective of choice of initial random control variables. Further for test case- 2 variations between best, mean and worst cost are less and are superior to pattern search methods. Figure 1, shows the convergence characteristics.

For both test cases the best cost is arrived after 440 to 450 objective function computations. It can also be observed that after 100 function evaluations the quality of objective function improves. Random search tool box arrived at best solutions after 350 to 470 objective function evaluations.

\section{Conclusion}

BB-BC optimization method is applied to solve OPF involving cost functions with valve point loading effects with continuous as well as mixed-integer type control variables. Simulation results are compared with commercially available pattern search MATLAB tool box optimization. Summary results of OPF indicates the effectiveness of BB$\mathrm{BC}$ in solving complex static OPF problems.A ten run statistical results indicates the reliability of BB-BC. Due to absence of tuning parameters in update equation of BB-BC it is numerically simple and yet can provide quality solutions to complex optimal Power flow.

\section{References}

[1] M.Huneault and F D Galiana 'A survey of the optimal Power Flow Literature'.IEEE Trans.vol 6, no, May 1991.

[2] Bansal 'optimization methods for Electrical power systems an overview' International journal of Emerging Electrical Power Systems' vol 2,Iss 1,Article 1021 available @ http://www.bepress.com//ijeeps/vol2/iss1/art 1021. 
[3] N.Sinha ,R.Chakrabarti,P.K.Chattopadhaya,'Evolutionary programming techniques for Economic load Dispatch'IEEE Trans.Evol.comp(1)(2003),pp 84-89.

[4] N.P.Padhy,'Artificial Intelligence and Intelligent Systems' Oxford University press.

[5] K. Erol Osman, Ibrahim Eksin, "New optimization method : Big Bang-Big Crunch",Elsevier, Advances in Engineering Software 37 (2006), pp. 106-111. www.mathworks.com.

[6] H.Yang and C.Huang,' Evolutionary Programming Based Dispatch for Units with Non-smooth Fuel cost functions 'IEEE Transactions on PWRS ,vol 11,no 1,February 1996,pp 112-118.

[7] Anatasis G.Balirtzis,N.Biskas,Christoforos E.Zoumas and Vasilios Petridis 'Optimal Power Flow by Enhanced Genetic Algorithms', IEEE Trans vol 17 no 2,May 2002 pp 229-236.

[8] P. H. Hota, R. Chakrabarti, P. K. Chattopadhya 'Multiobjective Dispatch Using Goal- Attainment Method' Journal of the Institution of Engineers (India), vol 82, September 2001, pp122-127.

[9] K.y.Lee,Y.M Park and J L Oritiz “A unified approach to optimal real and reactive power Dispatch", System Research, IEEE transaction on PAS ,vol PAS-104,no 5, May 1985, pp 1147-53.

Table 1: OPF results of best control variables:

\begin{tabular}{|c|c|c|}
\hline \multicolumn{3}{|l|}{ Test Case -1} \\
\hline Variable & BB-BC & Pattern search \\
\hline $\mathrm{p}_{\mathrm{g} 1}$ & 2.2952 & 2.2952 \\
\hline $\mathrm{P}_{\mathrm{g} 2}$ & 1.1744 & 1.1765 \\
\hline$P_{g 3}$ & 1.1287 & 1.1267 \\
\hline$P_{g 4}$ & 0.7500 & 0.7500 \\
\hline$P_{g 5}$ & 2.0982 & 2.0982 \\
\hline$v_{\mathrm{g} 1}$ & 1.0150 & 1.0251 \\
\hline $\mathrm{v}_{\mathrm{g} 2}$ & 1.0563 & 1.0646 \\
\hline $\operatorname{vg}_{3}$ & 1.0334 & 1.0494 \\
\hline $\mathrm{v}_{\mathrm{g} 4}$ & 1.0174 & 1.0247 \\
\hline$v_{g 5}$ & 1.0506 & 1.0454 \\
\hline \multicolumn{3}{|l|}{ Test Case -2 } \\
\hline Variable & BB-BC & Pattern search \\
\hline$p_{g 1}$ & 1.9407 & 1.9232 \\
\hline $\mathrm{p}_{\mathrm{g} 2}$ & 0.4560 & 0.3964 \\
\hline$p_{g} 5$ & 0.2049 & 0.2149 \\
\hline $\mathrm{p}_{\mathrm{g} 8}$ & 0.1144 & 0.1804 \\
\hline $\mathrm{p}_{\mathrm{g} 11}$ & 0.1028 & 0.1001 \\
\hline$p_{g 13}$ & 0.1200 & 0.1225 \\
\hline $\mathrm{v}_{\mathrm{g} 1}$ & 1.0823 & 1.0623 \\
\hline $\mathrm{v}_{\mathrm{g} 2}$ & 1.0575 & 1.0390 \\
\hline$v_{\mathrm{g} 5}$ & 1.0268 & 1.0038 \\
\hline $\mathrm{v}_{\mathrm{g} 8}$ & 1.0312 & 1.0162 \\
\hline $\mathrm{v}_{\mathrm{g} 11}$ & 1.1000 & 1.0742 \\
\hline $\mathrm{v}_{\mathrm{g} 13}$ & 1.0834 & 1.0672 \\
\hline$t_{11}$ & 1.0400 & 1.0200 \\
\hline$t_{12}$ & 1.0050 & 0.9050 \\
\hline$t_{15}$ & 1.0100 & 0.9600 \\
\hline$t_{17}$ & 0.9500 & 0.9350 \\
\hline
\end{tabular}

*all values in pu 
Table 2: summary of OPF results

\begin{tabular}{|c|c|c|c|c|}
\hline \multicolumn{2}{|c|}{ Test case-1 } & \multicolumn{3}{|c|}{ Test case- 2} \\
\hline variable & BB-BC & $\begin{array}{l}\text { PATTERN } \\
\text { SEARCH }\end{array}$ & BB-BC & $\begin{array}{l}\text { PATTERN } \\
\text { SEARCH }\end{array}$ \\
\hline Cost $(\$ / h r)$ & 2138.6 & 2138.1309 & 916.34 & 918.4607 \\
\hline $\begin{array}{l}\text { Total real power } \\
\text { Generation(MW }\end{array}$ & 744.70 & 744.65 & 293.88 & 293.75 \\
\hline $\begin{array}{l}\text { Total real losses } \\
\text { (MW) }\end{array}$ & 14.70 & 14.65 & 10.48 & 10.15 \\
\hline $\mathrm{V}_{\mathrm{MIN}}(\mathrm{pu})$ & $\begin{array}{l}0.98 \\
@ \text { bus }\end{array}$ & $\begin{array}{l}0.98 \\
@ \text { bus } 15\end{array}$ & $\begin{array}{l}1.02 \\
@ \text { bus? }\end{array}$ & $\begin{array}{l}1.01 \\
@ \text { bus } 30\end{array}$ \\
\hline $\mathrm{V}_{\mathrm{MAX}}(\mathrm{pu})$ & $\begin{array}{l}1.05 \\
@ \text { bus } 1\end{array}$ & $\begin{array}{l}1.05 \\
@ \text { bus } 10\end{array}$ & $\begin{array}{l}1.05 \\
\text { @bus: }\end{array}$ & $\begin{array}{l}1.05 \\
@ \text { @us } 27\end{array}$ \\
\hline $\begin{array}{l}V_{\text {MEAN }}(\mathrm{pu}) \\
\text { Thermal limit of }\end{array}$ & $\begin{array}{l}1.02 \\
120 \mathrm{MI}\end{array}$ & $\begin{array}{l}1.02 \\
\text { W line } 16\end{array}$ & $\begin{array}{l}1.03 \\
130 \mathrm{MV}\end{array}$ & $\begin{array}{l}1.03 \\
\mathrm{~A} \quad \text { line } 1\end{array}$ \\
\hline MAX flow(pu) & $\begin{array}{l}92.91 \\
\text { in line }\end{array}$ & $\begin{array}{l}92.61 \\
\text { in line } 16\end{array}$ & $\begin{array}{l}129.98 \\
\text { in line }\end{array}$ & $\begin{array}{l}130.0 \\
\text { In line } 1\end{array}$ \\
\hline
\end{tabular}

Table 3: Mean, worst and best cost in $(\$ / \mathrm{hr})$ with different initial control variables

\begin{tabular}{|c|c|c|c|c|c|c|}
\hline Method & & system -1 & & & st system-2 & \\
\hline BB-BC & $\begin{array}{c}\text { Mean } \\
2154.6960\end{array}$ & $\begin{array}{c}\text { Worst } \\
2287.3479\end{array}$ & best & $\begin{array}{l}\text { Mean } \\
916.7758\end{array}$ & $\begin{array}{c}\text { Worst } \\
917.5922\end{array}$ & best \\
\hline 2138.6873 & & & & 916.3428 & & \\
\hline $\begin{array}{l}\text { Pattern search } \\
2138.1309\end{array}$ & 2205.5860 & 2472.6723 & & $\begin{array}{l}923.7506 \\
918.4607\end{array}$ & 937.272 & \\
\hline
\end{tabular}

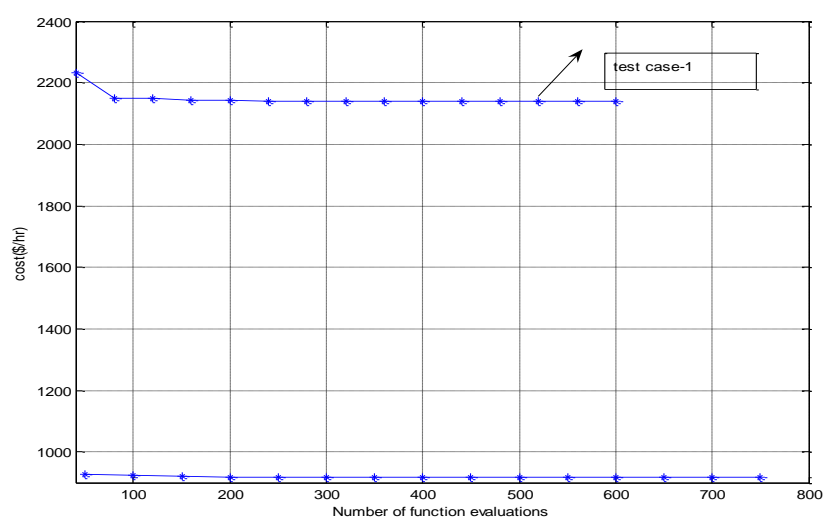

Figure 1: convergence characteristics of two test cases. 


\section{Appendix}

Generator cost curve coefficients and real power generation limits for Test System -1.

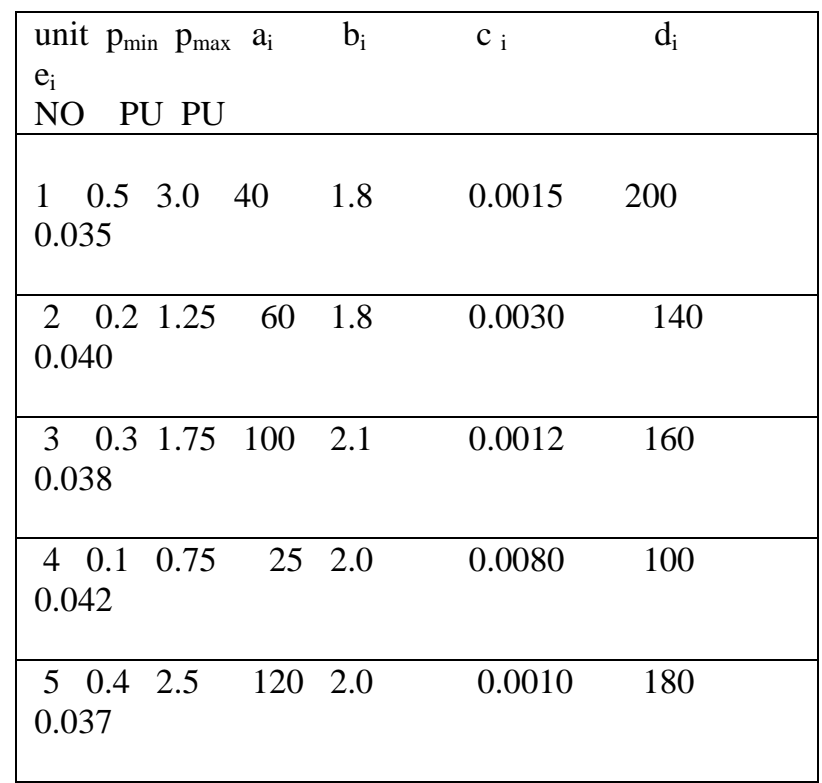

Generator cost curve coefficients and real power generation limits for Test System -2.

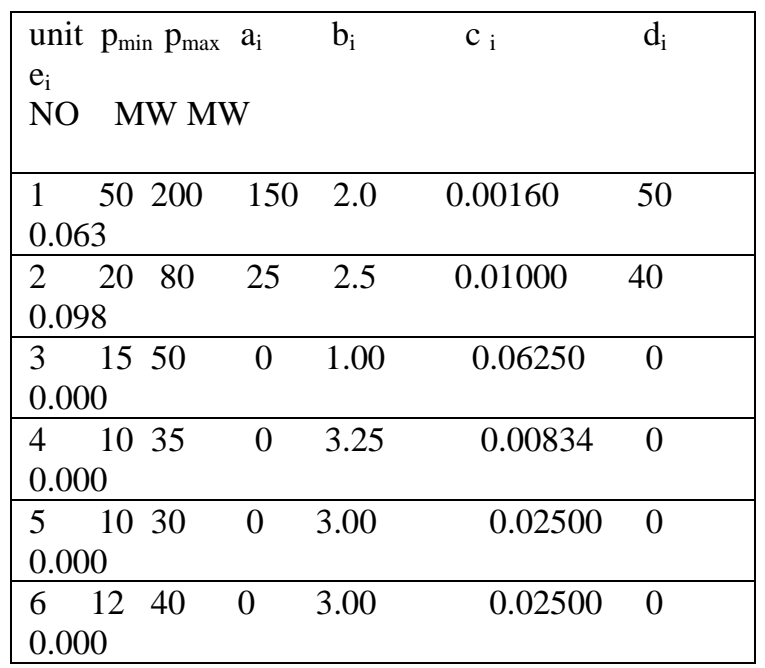

